\title{
Spin Dodecamer Formation in the Double-Exchange Spin Ice Model
}

\author{
Yoshihiro Shimomura*, Shin Miyahara and Nobuo Furukawa \\ Department of Physics and Mathematics, Aoyama Gakuin University, Sagamihara 229-8558, Japan
}

(Received December 11, 2018)

\begin{abstract}
We investigated the double-exchange spin ice (DESI) model on a kagomé lattice by Monte Carlo simulation to study the effects of a geometrical frustration, and the mechanism that generates an ordered state in a metallic system. The DESI model on the kagomé lattice is a frustrated metallic system due to an effective ferromagnetic interaction between localized spins caused by the double-exchange (DE) mechanism and a uniaxial anisotropy for the localized spins. A dodecagonal spin cluster (named dodecamer), which consists of twelve localized spins, appears at low temperature when the number of particles per site $n \simeq$ $1 / 3 \sim 1 / 2$. Such a dodecamer order is driven by both the kinetic energy gain due to the DE mechanism and the geometrical frustration. We discuss that cluster orders, in general, may be a common feature in itinerant electron systems coupled with frustrated adiabatic fields.

KEYWORDS: dodecamer, cluster order, double-exchange model, kagomé lattice, frustration, metal, spin ice, Monte Carlo simulation
\end{abstract}

Geometrically frustrated systems have been an attractive subject due to their novel phenomena after the predictions of disordered ground states in antiferromagnetic (AF) classical spin systems on geometrically frustrated lattices, i.e., a triangular, ${ }^{1}$ a kagomé, ${ }^{2,3}$ and a pyrochlore lattice. ${ }^{4}$ These disordered systems have macroscopically degenerate ground states accompanied with a finite residual entropy. Such a degeneracy can be lifted by additional effects, which leads to a novel ground state. For example, in frustrated quantum spin systems, ${ }^{5}$ quantum fluctuation sometimes lifts the degeneracy and induces a nonmagnetic ground state with a finite spin gap. One of the most well-known examples is a dimer singlet ground state observed in the Majumdar-Ghosh model ${ }^{6}$ and the Shastry-Sutherland model, ${ }^{7}$ which probably reflects the short-range interaction between localized spins.

The spin ice system in pyrochlore oxides ${ }^{8,9}$ provides us another insight on the lifting of the degeneracy. Localized spins in these systems have a strong uniaxial anisotropy in the $\langle 111\rangle$ direction for each corner-shared tetrahedron that constitutes the pyrochlore lattice. The interaction between nearest-neighbor (n.n.) spins is ferromagnetic. Each tetrahedron has the "two-in two-out" spin structure at low temperature, i.e., two spins point inward for the tetrahedron and the other two outward due to the uniaxial anisotropy and the ferromagnetic

\footnotetext{
*E-mail address: shimomura@phys.aoyama.ac.jp
} 
interaction. This is often called an ice rule after the positions of hydrogen atoms in the ice. According to recent researches, ${ }^{10-12}$ a long-range dipolar interaction generates a cluster order satisfying the ice rule, which cannot be explained by the n.n. ferromagnetic Ising interaction. This indicates that the long-range interaction lifts the degeneracy and creates a cluster order.

Considering these circumstances, it is highly significant to investigate various mechanisms for lifting the degeneracy due to the frustration. Naturally, frustrated electron systems are also expected to have a peculiar mechanism. As a candidate for electron systems, let us consider the double-exchange (DE) model. ${ }^{13}$ In this model, conduction electrons interact with localized spins through the on-site Hund's-rule coupling $J_{H}$, which produces the effective ferromagnetic interaction between n.n. localized spins. However, the range of interaction between localized spins is determined by the kinetics of electrons. ${ }^{14}$ In the strong Hund's-rule coupling limit $\left(J_{H} \rightarrow \infty\right)$, spins of conduction electrons are parallel to localized spins and ferromagnetic domains are formed at low temperature. Since electrons are confined within the domains, the electronic energy strongly depends on the sizes and the shapes of the domains. Therefore, in order to describe the energy of the system as a function of spin configuration, it is necessary to take into account effective long-range two- and/or multiple-spin interactions in each domain. Note that this is in contrast to the spin systems with the short-range interaction, where the energy depends on surface volumes of domain boundaries. In this way, frustrated DE systems may have unique features with respect to the mechanisms for lifting the degeneracy due to the kinetics of electrons.

Following this idea, we have constructed the double-exchange spin ice (DESI) model on a kagomé lattice, as an example of frustrated DE models. The model has an effective ferromagnetic interaction due to the DE mechanism and a uniaxial anisotropy for localized spins as in the spin ice system. Therefore, this system has the frustration. In this study, we have investigated the low-temperature behavior of the DESI model using the Monte Carlo (MC) method. From MC calculations, we obtain the following: (1) A dodecagonal localized spin cluster, "dodecamer", is realized at low temperature, (2) the dodecamer phase exists in a wide doping region $n \simeq 1 / 3 \sim 1 / 2$, where $n$ is the number of particles per site, and (3) the dodecamer order is driven by both the kinetic energy gain due to the DE mechanism and the frustration. On the analogy of the DESI system on the kagomé lattice, similar cluster orders might be generic features in frustrated electron systems.

Let us start from the DE model in the strong Hund's-rule coupling limit ${ }^{13,15}$

$$
H=-\sum_{\langle i, j\rangle}\left\{\tilde{t}\left(\vec{S}_{i}, \vec{S}_{j}\right) \tilde{c}_{i}^{\dagger} \tilde{c}_{j}+\text { h.c. }\right\}-\mu \sum_{i} \tilde{c}_{i}^{\dagger} \tilde{c}_{i},
$$

where $\tilde{c}_{i}^{\dagger}\left(\tilde{c}_{i}\right)$ is an electron operator that creates (annihilates) an electron at site $i$ with a spin parallel to a localized spin $\vec{S}_{i}$. The effective hopping matrix element is defined as $\tilde{t}\left(\vec{S}_{i}, \vec{S}_{j}\right)=$ $t \cos \left(\theta_{i j} / 2\right) \exp \left(i \gamma_{i j}\right)$, where $t$ represents the n.n. transfer integral, $\theta_{i j}$ is an angle between $\vec{S}_{i}$ 


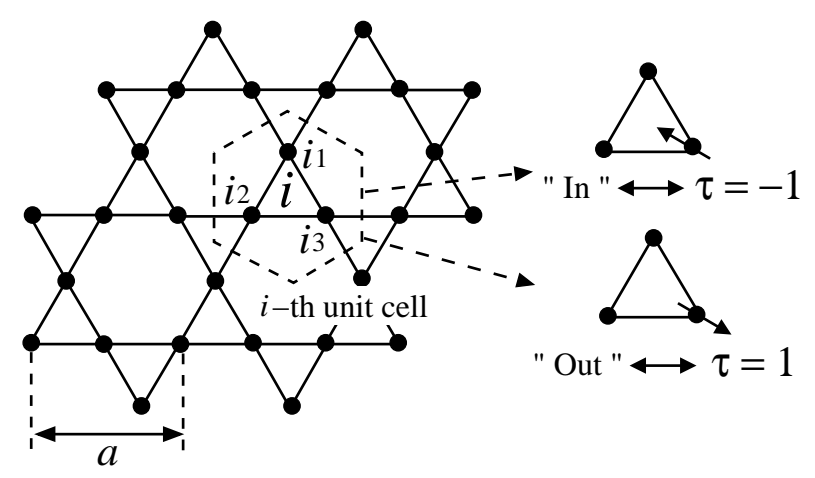

Fig. 1. DESI model on the kagomé lattice. The dashed line is an $i$-th unit cell composed of three sites $\left(i_{1}, i_{2}\right.$ and $\left.i_{3}\right)$, and $a$ is a lattice constant. The direction of a localized spin is shown by arrows: inward (in-spin) or outward (out-spin) for an up-triangle in the unit cell. $\tau$ is a pseudo-spin operator, where an out-(in-)spin corresponds to a pseudo-up (down) spin.

and $\vec{S}_{j}$, and $\gamma_{i j}$ is a phase factor that creates the Berry phase at the noncoplanar configuration of spins. Hereafter, we set that $t$ is positive, and localized spins are classical ones for simplicity. Note that the absolute value of $\tilde{t}\left(\vec{S}_{i}, \vec{S}_{j}\right)$ becomes a maximum when $\vec{S}_{i}$ and $\vec{S}_{j}$ are parallel, which leads to the effective ferromagnetic interaction between the n.n. spins to gain the kinetic energy locally. However, as described above, the electronic energy is determined by the kinetics of electrons, which depends on the sizes and shapes of ferromagnetic domains where electrons move easily. Thus, it is found that effective multiple interactions are important to determine the nature of the DE system.

In this letter, we consider the DE model on the kagomé lattice and assume a uniaxial anisotropy for the localized spin, which is forced to point inward (in-spin) or outward (outspin) for an up-triangle in a unit cell of the kagomé lattice [see Fig. 1]. Spins are oriented within the kagomé plain, i.e., coplanar under this constraint. We consider the model without the phase factor $\gamma_{i j}$ in $\tilde{t}\left(\vec{S}_{i}, \vec{S}_{j}\right)$. An in-spin is energetically preferable next to an out-spin on each triangle due to the DE mechanism. This situation is similar to the spin ice system..$^{8,9}$ Therefore, we call the model the DESI model. We define a pseudospin at site $i$,

$$
\tau_{i}= \begin{cases}+1 & \left(\vec{S}_{i} \text { is an out-spin }\right) \\ -1 & \left(\vec{S}_{i} \text { is an in-spin }\right)\end{cases}
$$

In this representation, the effective ferromagnetic interaction between n.n. localized spins is regarded as the AF interaction between n.n. pseudospins. Thus, it is naturally considered that each triangle has a "two-down one-up" or "one-down two-up" pseudospin structure at low temperature, which corresponds to a "two-in one-out" or "one-in two-out" localized spin structure, respectively. Hereafter, we use the pseudospin picture $\left\{\tau_{i}\right\}$ instead of the bare localized spin picture $\left\{\vec{S}_{i}\right\}$. One may think that the DESI model on the kagomé lattice can 


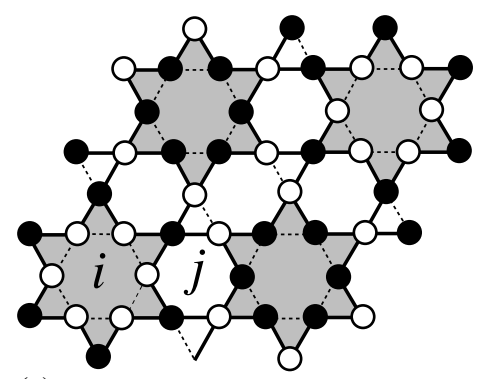

(a)

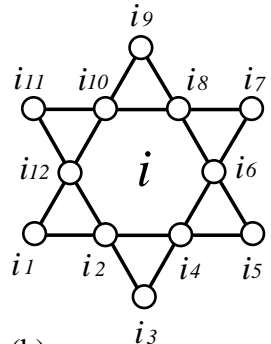

(b)

Fig. 2. (a) Snapshot of localized spins at $k_{B} T / t=0.01$ and $\mu / t=0$. In-(out-)spins are represented by open (closed) circles. An in-spin and an out-spin are connected by a solid line, while the nearestneighbor pairs of in-(out-)spins are connected by a dashed line. Electrons can move easily through solid lines. Each hexagon is labeled $i, j$ and others. The shadowed dodecagonal spin cluster is a dodecamer. (b) A dodecamer consists of twelve localized spins. Labels $i_{1}, \ldots, i_{12}$ are site numbers on the $i$-th dodecamer.

be mapped onto the AF Ising model on the same lattice, which has a disordered ground state with a macroscopic degeneracy. ${ }^{2,3}$ However, the nature of the former system should be determined by the kinetics of electrons, i.e., effective long-range interaction, and it is expected that the behavior of the DESI model may be quite different from the frustrated Ising spin system with the short-range interaction.

We have performed MC calculations ${ }^{16}$ to study the thermodynamics of the system described by the Hamiltonian (1) at finite temperatures. We typically run 100,000 MC steps for measurement after 10,000 thermalization steps. We applied the Metropolis algorithm for the updates of spin configurations. ${ }^{17}$

In order to investigate the features of the localized spins, we have calculated a pseudospin structure factor defined as

$$
T_{q}^{\alpha \beta}=\frac{1}{N_{\mathrm{c}}} \sum_{i, j} e^{\mathrm{i} \vec{q} \cdot\left(\vec{r}_{i_{\alpha}}-\vec{r}_{j_{\beta}}\right)}\left\langle\tau_{i_{\alpha}} \tau_{j_{\beta}}\right\rangle,
$$

where $N_{\mathrm{c}}$ is the number of unit cells on the kagomé lattice, $i_{\alpha}$ and $i_{\beta}$ ( $\alpha$ and $\beta=1$, 2 , or 3 ) indicate three sites in an $i$-th unit cell shown in Fig. 1, and the summation for $i$ and $j$ extends over all unit cells. $\langle\cdots\rangle$ represents the thermal average. Given the chemical potential $\mu / t=0$ and $N_{\mathrm{c}}=4 \times 4$ (= $=48$ sites $)$ and $8 \times 8$ (=192 sites), results at sufficiently low temperature $\left(k_{B} T / t=0.01\right)$ are as follows: (1) Maximum eigenvalues of $T_{q}^{\alpha \beta}$ are observed at $\vec{q}_{1}={ }^{t}(3 \pi / 2 a, \pi / 2 a)$ and $\vec{q}_{2}={ }^{t}(\pi / 2 a, 3 \pi / 2 a)$. (2) Corresponding eigenmodes represent a two-in one-out or one-in two-out spin structure. These results mean that the translational symmetry of the lattice breaks at low temperature. Such a symmetry breaking is also observed simultaneously in a snapshot of localized spins [see Fig. 2(a)], and the periodicity of spins is almost consistent with that represented by $\vec{q}_{1}$ and $\vec{q}_{2}$. Note that there are two kinds of bond: 


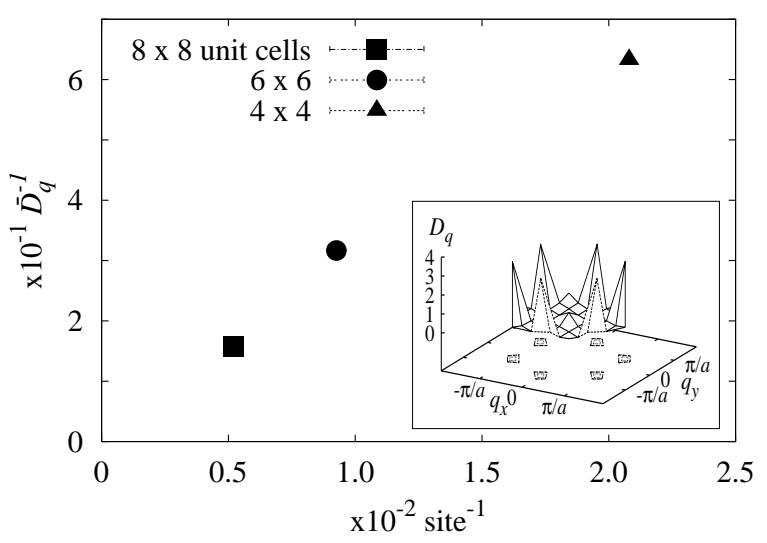

Fig. 3. System size dependence of $\bar{D}_{q}$ at $k_{B} T / t=0.006$ and $\mu / t=0$, where $\bar{D}_{q} \equiv\left\{D_{Q_{1}}+D_{Q_{2}}+\right.$ $\left.D_{Q_{3}}\right\} / 3$.,$\bullet$, and $\boldsymbol{\Delta}$ represent $8 \times 8$ (= 192 sites), $6 \times 6$ (=108 sites) and $4 \times 4$ (= 48 sites) unit cells, respectively. Error bars are within the sizes of the symbols. It is found that $\bar{D}_{q}$ grows proportional to $N_{\mathrm{c}}$. The inset is the dodecamer structure factor with the lattice size $N_{\mathrm{c}}=6 \times 6$.

a bond between in- and out-spins [solid line in Fig. 2(a)] and that between same spins [dashed line in Fig. 2(a)]. Electrons can easily move through the former bond due to a larger $\tilde{t}\left(\vec{S}_{i}, \vec{S}_{j}\right)$ value. Connecting bonds of the former type, the kagomé lattice can be tiled by the dodecagonal spin cluster which we call a "dodecamer" after twelve localized spins, as shown in Fig. 2(a).

The existence of the dodecamers can be read off using an order parameter defined by

$$
d_{i} \equiv \frac{1}{18}\left(-\sum_{n=1}^{12} \tau_{i_{n}} \tau_{i_{n+1}}+\sum_{m=1}^{6} \tau_{i_{2 m}} \tau_{i_{2 m+2}}\right),
$$

where a site $i_{n}(n=1,2, \cdots, 12)$ is indicated in Fig. $2(\mathrm{~b})$ and we set $\tau_{i_{13}}=\tau_{i_{1}}$ and $\tau_{i_{14}}=\tau_{i_{2}}$ from the periodicity of the lattice. Note that the order parameter $d_{i}$ is a unity when the dodecamer exists. The structure factor of a correlation function $\left\langle d_{i} d_{j}\right\rangle$ is defined by

$$
D_{q}=\frac{1}{N_{\mathrm{c}}} \sum_{i, j} e^{\mathrm{i} \vec{q} \cdot\left(\vec{r}_{i}-\vec{r}_{j}\right)}\left\langle d_{i} d_{j}\right\rangle,
$$

where $\sum$ represents the summation for any pairs of the order parameters shown in Fig. 2 (a).

The results with the lattice size $N_{\mathrm{c}}=6 \times 6$ at $k_{B} T / t=0.006$ and $\mu / t=0$ are shown in the inset of Fig. 3. There are three independent peaks at $\vec{Q}_{1}={ }^{t}(0,2 \pi / \sqrt{3} a), \vec{Q}_{2}={ }^{t}(\pi / a, \pi / \sqrt{3} a)$ and $\vec{Q}_{3}={ }^{t}(\pi / a,-\pi / \sqrt{3} a)$, which is consistent with the periodicity of the dodecamer order state shown in Fig. 2 (a).

The system size dependence of the averaged structure factor $\bar{D}_{q} \equiv\left\{D_{Q_{1}}+D_{Q_{2}}+D_{Q_{3}}\right\} / 3$ for $N_{\mathrm{c}}=4 \times 4,6 \times 6$ and $8 \times 8$ is shown in Fig. 3. The result indicates that the structure factor is proportional to the system size $N_{\mathrm{c}}$. Thus, we concluded that the dodecamer order survives even in the thermodynamic limit.

To see the stability of the dodecamer order, the temperature dependence of $\bar{D}_{q}$ with various 


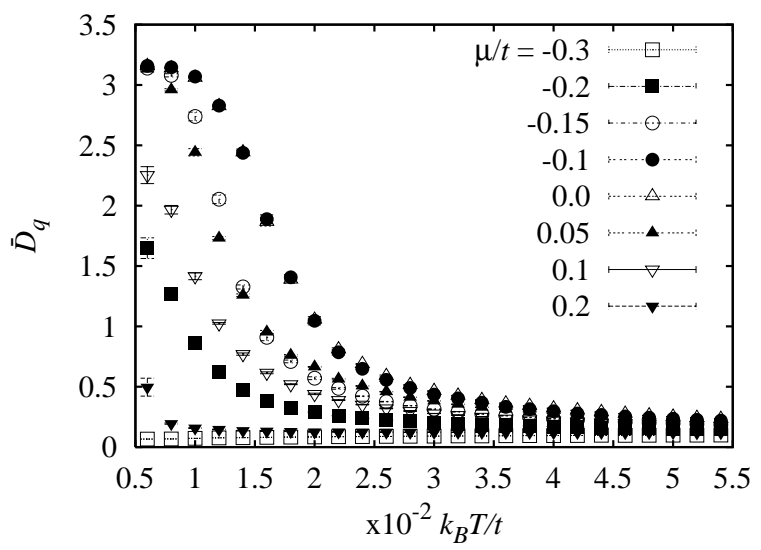

Fig. 4. Temperature dependence of $\bar{D}_{q}$ at $\mu / t=-0.3(\square),-0.2(\boldsymbol{\square}),-0.15(\circ),-0.1(\bullet), 0.0(\triangle), 0.05$ $(\mathbf{\Delta}), 0.1(\nabla)$ and $0.2(\boldsymbol{\nabla})$ when the system size $N_{\mathrm{c}}$ is $6 \times 6(=108$ sites $)$.

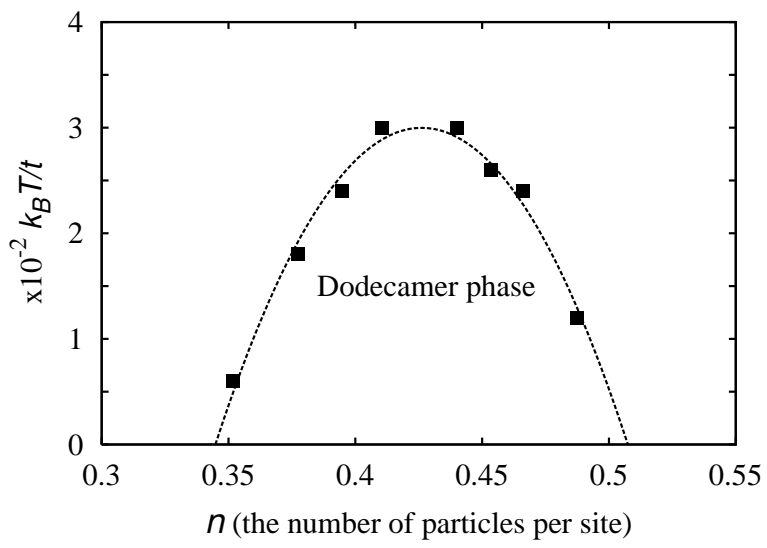

Fig. 5. Estimated phase diagram with $N_{\mathrm{c}}=6 \times 6$ (=108 sites) as a function of the number of particles per site $n$ and the temperature. The dodecamer phase can exist in the region $n \simeq 1 / 3 \sim 1 / 2$.

chemical potentials $(\mu / t=-0.3,-0.2,-0.15,-0.1,0.0,0.05,0.1$ and 0.2$)$ at $k_{B} T / t \leq 0.054$ has been calculated. As shown in Fig. 4 , several $\bar{D}_{q}$ 's grow rapidly at low temperature, which roughly indicates a phase transition from the disordered state to the dodecamer state. Note that the constraints for localized spins, i.e., two-in one-out or one-in two-out, are satisfied in this temperature range. We define the pseudotransition temperature from the deviation of the data in Fig. 4 from the Curie-Weiss law. Such a transition temperature is not rigorous but at least gives an upper limit. An estimated phase diagram is summarized in Fig. 5. We expect that the dodecamer order exists in the doping region $n \simeq 1 / 3 \sim 1 / 2(-0.3 \lesssim \mu \lesssim 0.2)$.

Let us consider the origin of the dodecamer order state. We consider a few scenarios: (1) the formation of an electron hopping path where the kinetic energy of electrons can be gained in terms of the DE mechanism and (2) the stabilization caused by an energy gap around 


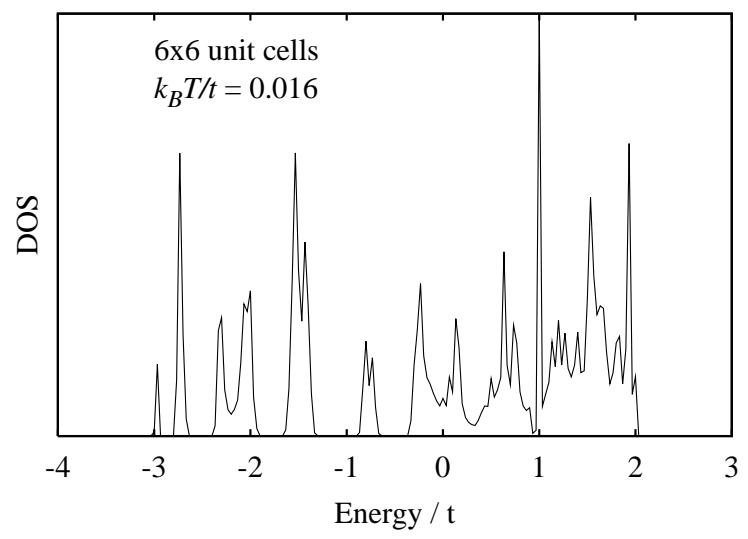

Fig. 6. Density of states (DOS) at $N_{\mathrm{c}}=6 \times 6(108$ sites $)$ and $k_{B} T / t=0.016$. The dodecamer order appears at $-0.3 \lesssim \mu \lesssim 0.2$.

the Fermi level breaking the translational symmetry of the lattice in some manners. The former case corresponds to a metallic state, and the latter case, an insulating state. To clarify this point, we have investigated the density of states (DOS). The DOS at $N_{\mathrm{c}}=6 \times 6$ and $k_{B} T / t=0.016$ is shown in Fig. 6 . The result indicates that the energy gap is not opened around the chemical potentials $(-0.3 \lesssim \mu / t \lesssim 0.2)$ when the dodecamer state is realized, which means that the dodecamer state corresponds to a metallic state in terms of the conventional band picture. This result excludes the latter possibility and is reasonable, because the DESI model originally has the only DE mechanism that prevents insulating states. This is also consistent with the result that the stability of the dodecamer order is not sensitive to the shape of the Fermi surface indicated by the existence of the wide doping region for the dodecamer state. Note that the two-in one-out or one-in two-out structure has been maintained in the dodecamer state. This finding indicates that the ferromagnetic interaction, that is, the DE mechanism due to the metallic nature of electrons is important even in the dodecamer phase. This strongly indicates that the dodecamer cluster is driven by both the frustration and the kinetic energy gain due to the DE mechanism.

In conclusion, the dodecamer order appears at low temperature in the DESI model on the kagomé lattice because of the kinetic energy gain due to the DE mechanism and the frustration of the model. The DESI model can be realized in other lattices, for example, the pyrochlore lattice. Thus, it is natural to expect the cluster order state in the DESI model not only on the kagomé lattice but also on the pyrochlore lattice. Cluster orders may exist in real materials which have the pyrochlore structure, the DE mechanism, and the frustration. One of the possibilities is that in a pyrochlore oxide $R_{2} \mathrm{Mo}_{2} \mathrm{O}_{7}$, where $R$ is a trivalent rareearth ion $(\mathrm{Nd}, \mathrm{Sm}, \mathrm{Gd}, \mathrm{Tb}, \mathrm{Dy}, \mathrm{Yb})$ and $\mathrm{Y} .{ }^{18-22}$ There is a crossover from the ferromagnetic metallic (FM) phase to the spin-glass ( $\mathrm{SG}$ ) phase as the mean ionic radius $R$ decreases, i.e., 
the bandwidth control. ${ }^{20,21}$ Since the magnetoresistance effect supports that the FM state is caused by the DE mechanism between $d$ electrons on $\mathrm{Mo}^{4+}$, this series might be a realization of the DESI model due to both the geometry of the pyrochlore lattice and the strong anisotropy of spins. In particular, the FM state near the phase boundary has a character of the SG state indicated by ordinary and anomalous Hall coefficients ${ }^{20}$ and an elastic neutron scattering measurement also indicates a short-range spin order in $\mathrm{Y}_{2} \mathrm{Mo}_{2} \mathrm{O}_{7},{ }^{23}$ which may be explained by considering cluster orders. If the uniaxial anisotropy of spins in our model is weakened, i.e., spins change from Ising to Heisenberg, it is obvious that the system has the FM ground state, which corresponds to $\mathrm{Nd}_{2} \mathrm{Mo}_{2} \mathrm{O}_{7}$. In this way, we may explain the transition changing the strength of the uniaxial anisotropy and anomalous behaviors observed in this series.

In terms of cluster formation in frustrated systems, the effective interaction due to the kinetics of electrons in the DESI system corresponds to the long-range dipolar interaction in the spin ice system. Thus, we expect that the long-range nature is important to create cluster orders in both frustrated spin and electron systems. Although two-body (short-range) interactions, such as the n.n. Ising interaction, are usually sufficient to create ordered states in nonfrustrated systems, these interactions are not sufficient to lift the degeneracy in the frustrated systems. Thus, other effects, which can be neglected in conventional nonfrustrated systems, are important in frustrated systems. In the DESI model, the kinetic energy gain corresponds to such effects. In other frustrated electron systems, the formation of similar clusters where electrons can move easily is also expected to gain the kinetic energy. For example, an electron-lattice coupled system is the case, where we may find the lattice-distorted cluster. Even in frustrated systems with the short-range electron-electron interaction such as the Hubbard model, similar cluster orders can exist in the same manner. Since studies in the Hubbard model on the frustrated lattice are limited, ${ }^{24}$ further developments, particularly apart from the half-filling case, are desired.

We would like to thank H. Tsunetsugu and N. Kawakami for helpful suggestions. The authors also thank M. Tsutsui and S. Katsurada for computational support. The numerical computations have been performed mainly using the facilities of the AOYAMA+ project. This work was partially supported by a Grant-in-Aid for 21st COE program from the Ministry of Education, Culture, Sports, Science and Technology of Japan. 


\section{References}

1) G. H. Wannier: Phys. Rev. 79 (1950) 357.

2) I. Syozi: Prog. Theor. Phys. 6 (1951) 306.

3) K. Kano and S. Naya: prog. Theor. Phys. 10 (1953) 158.

4) P. W. Anderson: Phys. Rev. 102 (1956) 1008.

5) G. Misguich and C. Lhuillier: cond-mat/0310405, and references therein.

6) C. K. Majumdar and D. K. Ghosh: J. Math. Phys. 10 (1969) 1399.

7) B. Shastry and B. Sutherland: Physica B 108 (1981) 1069.

8) M. J. Harris, S. T. Bramwell, D. F. McMorrow, T. Zeiske and K. W. Godfrey: Phys. Rev. Lett. 79 (1997) 2554.

9) A. P. Ramirez, A. Hayashi, R. J. Cava, R. Siddharthan and B. S. Shastry: Nature (London) 399 (1999) 333.

10) B. C. den Hertog and M. J. P. Gingras: Phys. Rev. Lett. 84 (2000) 3430.

11) S. T. Bramwell, M. J. Harris, B. C. den Hertog, M. J. P. Gingras, J. S. Gardner, D. F. McMorrow, A. R. Wildes, A. L. Cornelius, J. D. M. Champion, R. G. Melko and T. Fennell: Phys. Rev. Lett. 87 (2001) 047205.

12) R. G. Melko, B. C. den Hertog and M. J. P. Gingras: Phys. Rev. Lett. 87 (2001) 67203.

13) C. Zener: Phys. Rev. 82 (1951) 403.

14) Y. Motome and N. Furukawa: J. Phys. Soc. Jpn. 70 (2001) 1487.

15) P. W. Anderson and H. Hasegawa: Phys. Rev. 100 (1955) 675.

16) S. Yunoki, J. Hu, A. L. Malvezzi, A. Moreo, N. Furukawa and E. Dagotto: Phys. Rev. Lett. 80 (1998) 845.

17) N. Metropolis, A. W. Rosenbluth, M. N. Rosenbluth, A. M. Teller and E. Teller: J. Chem. Phys. 21 (1953) 1087.

18) J. E. Greedan, M. Sato, N. Ali and W. R. Datars: J. Solid State Chem. 68 (1987) 300.

19) N. Ali, M. P. Hill and S. Labroo: J. Solid State Chem. 83 (1989) 178.

20) T. Katsufuji, H. Y. Hwang and S.-W. Cheong: Phys. Rev. Lett. 84 (2000) 1998.

21) Y. Moritomo, Sh. Xu, A. Machida, T. Katsufuji, E. Nishibori, M. Takata, M. Sakata and S.-W. Cheong: Phys. Rev. B 63 (2001) 144425.

22) Y. Taguchi, Y. Oohara, H. Yoshizawa, N. Nagaosa and Y. Tokura: Science 291 (2001) 2573.

23) J. S. Gardner, B. D. Gaulin, S.-H. Lee, C. Broholm, N. P. Raju and J. E. Greedan: Phys. Rev. Lett. 83 (1999) 211.

24) For example, on the kagomé lattice, Y. Imai and N. Kawakami: Phys. Rev. B 68 (2003) 195103, and references therein. 